\title{
Large Dosage of Chishao in Formulae for Cholestatic Hepatitis: A Systematic Review and Meta-Analysis
}

\author{
Xiao Ma, ${ }^{1,2}$ Ji Wang, ${ }^{3}$ Xuan He, ${ }^{4}$ Yanling Zhao, ${ }^{2}$ Jiabo Wang, ${ }^{2}$ Ping Zhang, ${ }^{5}$ Yun Zhu, \\ Lin Zhong, ${ }^{1,2}$ Quanfu Zheng, ${ }^{1,2}$ and Xiaohe Xiao ${ }^{2}$ \\ ${ }^{1}$ Pharmacy College, Chengdu University of Traditional Chinese Medicine, Chengdu 611137, China \\ ${ }^{2}$ China Military Institute of Chinese Medicine, 302 Hospital of People's Liberation Army, Beijing 100039, China \\ ${ }^{3}$ Department of Evidence-Based Medicine and Clinical Epidemiology, West China Hospital, Sichuan University, \\ Chengdu 610041, China \\ ${ }^{4}$ State Key Laboratory of Biotherapy, West China Hospital, Sichuan University, Chengdu 610041, China \\ ${ }^{5}$ Department of Integrative Medical Center, 302 Hospital of People's Liberation Army, Beijing 100039, China
}

Correspondence should be addressed to Yanling Zhao; zhaoyl2855@126.com and Xiaohe Xiao; xiaoxiaohe302@126.com

Received 3 April 2014; Accepted 15 May 2014; Published 2 June 2014

Academic Editor: Zunjian Zhang

Copyright (C) 2014 Xiao Ma et al. This is an open access article distributed under the Creative Commons Attribution License, which permits unrestricted use, distribution, and reproduction in any medium, provided the original work is properly cited.

Objective. To evaluate the efficacy and safety of large dosage of Chishao in formulae for treatment of cholestatic hepatitis. Methods. The major databases (PubMed, Embase, Cochrane Library, Chinese Biomedical Database Wanfang, VIP medicine information system, and China National Knowledge Infrastructure) were searched until January 2014. Randomized controlled trials (RCTs) of large dosage of Chishao in formulae that reported on publications in treatment of cholestatic hepatitis with total efficacy rate, together with the biochemical indices including alanine aminotransferase (ALT), aspartate aminotransferase (AST), total bilirubin (TBIL), and direct bilirubin (DBIL), were extracted by two reviewers. The Cochrane tool was used for the assessment of risk of bias included trials. Data were analyzed with RevMan 5.2.7 software. Results. 11 RCTs involving 1275 subjects with cholestatic hepatitis were included. Compared with essential therapy, large dosage of Chishao in formulae demonstrated more efficiently with down regulation of serum ALT, AST, TBIL, DBIL. Meanwhile, there were no obvious adverse events. Conclusion. As a promising novel treatment approach, widely using large dosage of Chishao in formulae may enhance the curative efficacy for cholestatic hepatitis. Considering being accepted by more and more practitioners, further rigorously designed clinical studies are required.

\section{Introduction}

Cholestatic hepatitis presents the symptoms as abnormal bile generation, secretion, and excretion that leads to the intrahepatic accumulation of bile acids (BA) and other toxic compounds with progression of liver pathology. It is one of the most common but devastating manifestations in many liver diseases, and without proper treatment, it will ultimately result in cirrhosis and hepatic failure $[1,2]$. Presently, there are 350 million to 400 million HBV infected individuals worldwide, and 2 percent of them suffer from cholestatic hepatitis along the progress $[3,4]$. Cholestatic hepatitis known for its severe and prolong jaundice characteristic brings heavy burden to patients and society [5]. Current therapy, aiming at symptoms and mechanism, generally takes several measures such as vitamin for adequate nutrition, albumin and plasma for supporting treatment, symptomatic treatment to cutaneous pruritus, ursodeoxycholic acid (UDCA) or adrenocortical hormone for removing jaundice, and even transplantation. However, several unsatisfactory aspects and side effects could be seen in clinics, and the alternative medicine or treatment is more necessary.

Along with development of medical science, complementary and alternative medicine displays its unique role in hepatitis treatment [6-8]. Traditional Chinese medicine (TCM), known for its holistic concept and treatment based on syndrome differentiation, emphasizes formulae employment more than single drug use in treatment of diseases. 
Increasing current studies demonstrate that formulae with definite efficacy for specific disease regulate function of multiple organs and tissues in a way of network $[9,10]$. In TCM, Yin Chen Hao Decoction has been widely used in treatment of jaundice. However, Yin Chen Hao Decoction usually fails in severe jaundice with the symptom of blood stasis. Chishao, owing to its function of blood invigoration, has been used in severe cholestatic hepatitis for decades and obtained satisfactory effects. Chishao, the dried root of Paeonia lactiflora pall or Paeonia veitchii Lynch, has been used for blood stasis and hyperactivity of liver-Yang in more than 2000 years. In 1983, Wang et al. proposed a novel approach of severe jaundice treatment by using large dosage of Chishao [11]. Following clinical studies, it suggested significant effect of Chishao in large dosage for cholestatic hepatitis [12]. Besides, Chishao in large dosage could ameliorate cholestasis induced by ANIT and bile duct ligation in animals [13]. By now, it demonstrates a promising treatment to use large dosage of Chishao on severe cholestasis in clinic. However, the systematic review on Chishao used in large dosage has not been seen. Therefore, this meta-analysis of RCTs was conducted to assess the clinical value of large dosage of Chishao relevant formulae for the treatment of cholestatic hepatitis.

\section{Materials and Methods}

2.1. Inclusion Criteria. The inclusion criteria were as follows. (1) Randomized controlled trials (RCTs) of patients diagnosed with cholestatic hepatitis met the criteria of Viral Hepatitis Prevention and Treatment Programs, Guidance for Clinical Research on New Drugs of TCM [25, 26]. (2) Chishao with large dose (more than $25 \mathrm{~g}, 2$ times larger than the prescribed maximum dose of Chinese Pharmacopeia 2010 version) serves as the main element in formulae alone or in combination with conventional therapy compared with placebo, conventional therapy as controls. (3) Outcome measurements included one or more indices as total efficacy rate, alanine aminotransferase (ALT), aspartate aminotransferase (AST), total bilirubin (TBIL), and direct bilirubin (DBIL).

2.2. Exclusion Criteria. The exclusion criteria were (1) reviews, nonclinical studies, and case observations, (2) no RCTs, and (3) controlled interventions with TCM therapies as other Chinese herbs or acupuncture.

2.3. Search Strategy. Comprehensive searches were both performed in English and Chinese databases including PubMed, Embase, Cochrane Library, Chinese Biomedical Database (CBM), Wanfang, VIP medicine information system (VMIS), and China National Knowledge Infrastructure (CNKI) from their inception to January 2014. Search terms included traditional Chinese medicine, Chishao, Paeonia lactiflora pall, Paeonia veitchii Lynch, large dose, hepatitis, cholestatic hepatitis, cholestasis, randomized controlled trial, and clinical controlled trial.
2.4. Data Extraction and Risk of Bias Assessment. Data extraction and quality assessment were independently performed by two researchers (Ma Xiao and He Xuan) and disagreements were resolved by consensus. Detailed data as study design, participants' information, interventions, outcome measures, and adverse event report were extracted to a conclusive table. Assessment of symptom improvement was based on Guidance for Clinical Research on New Drugs of TCM or Disease Diagnosis and Efficacy Standards of Chinese Internal Medicine.

Cochrane risk of bias tool was used to assess methodological quality of included RCTs. There are six domains including random sequence generation (selection bias), allocation concealment (selection bias), blinding of participants and personnel (performance bias), blinding of outcome data (attrition bias), incomplete outcome data (attrition bias), and selective reporting (reporting bias). The judgment was marked as "high risk," "unclear risk," or "low risk." Trials that met all the criteria were categorized as high risk of bias, whereas those that met none of the criteria were categorized as low risk of bias. The others were classified as unclear risk of bias if insufficient information was available to make a judgment.

2.5. Data Analysis. Statistical analysis was performed by Cochrane RevMan 5.2.7 (Cochrane Collaboration). Dichotomous data were presented as risk ratio (RR) and continuous variables as mean difference (MD), with $95 \%$ confidence intervals $(95 \% \mathrm{CI})$. Statistical heterogeneity was assessed by Cochrane's $Q$ test. Data with low heterogeneity $(P \geq 0.10$ and $\left.I^{2} \leq 50 \%\right)$ were performed as fixed-effects model whereas others performed as as random-effects model. A funnel plot was used for assessing the potential publication bias.

\section{Results}

3.1. Characteristics of Included Trials. A total of 1437 publications were identified for initial screen and 516 duplicated citations were removed. In the remaining 921 reports, 119 reviews/commentaries and 753 records irrelevant to the study were excluded. 49 full-text articles were used for further assessment. Among them, 18 nonrandomized controlled studies, 5 animal studies, 4 inconsistent criteria, 9 Chinese herbs in control group and 2 low dosage of Chishao were excluded. The rest of 11 eligible studies were conformed to meta-analysis (Figure 1).

11 articles involving 1275 subjects with cholestatic hepatitis (751 cases in trials group, 524 cases in control group) were included in this study, and there was no significance in ages, sex, and course of disease between two groups (Table 1). Essential treatment with large dose Chishao formulae or single Chishao formulae was used in trials group, whereas essential treatment or single western medicine was applied in control group. 10 of 11 articles described treatment duration that ranged from 30 days to 2 months [14, 16-24] and 4 reported follow-up period from 2 weeks to 6 months [21-24]. Six articles reported advent events with or without slight side effect [14, 18, 21-24] (Table 2). 


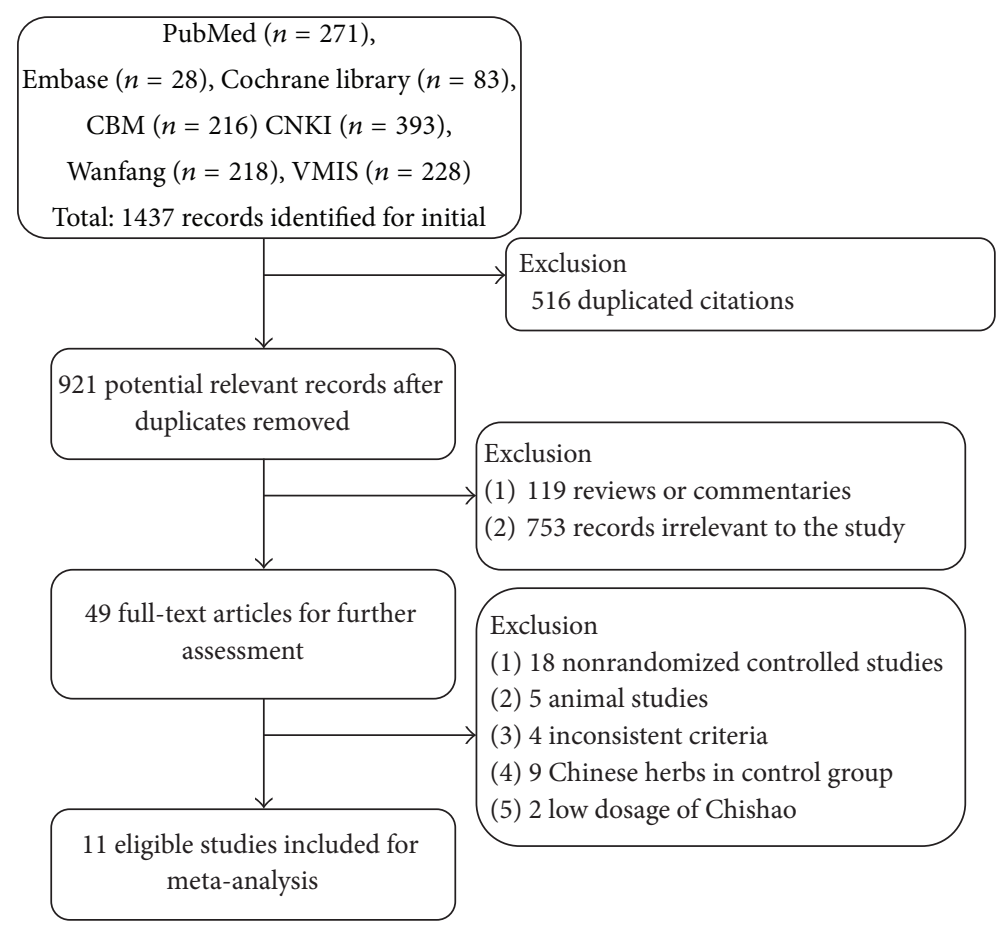

FIGURE 1: Flow diagram showing the trial selection process for the systematic review.

TABLE 1: Characteristics of included studies.

\begin{tabular}{|c|c|c|c|c|c|}
\hline Author (reference) & Published year & Cases T/C & $\begin{array}{l}\text { Age (years) } \\
\text { Range, mean }\end{array}$ & $\begin{array}{c}\text { Sex } \\
\text { Male/female }\end{array}$ & $\begin{array}{c}\text { Course of } \\
\text { disease (years) } \\
\text { Range, mean }\end{array}$ \\
\hline Tang et al. [14] & 2013 & $50 / 50$ & $\begin{array}{l}\mathrm{T}: 24-65,41.2 \\
\mathrm{C}: 23-67,42.1\end{array}$ & $\begin{array}{l}\text { T: } 37 / 13 \\
\text { C: } 38 / 12\end{array}$ & $\begin{array}{l}\mathrm{T}: 0-0.7,0.4 \\
\mathrm{C}: 0-0.8,0.4\end{array}$ \\
\hline Chen and $\mathrm{Tu}$ [15] & 2013 & $30 / 20$ & NR & $\begin{array}{l}\text { T: } 20 / 10 \\
\text { C: } 15 / 5\end{array}$ & NR \\
\hline Wu [16] & 2012 & $45 / 45$ & $\begin{array}{l}\mathrm{T}: 33-68,47.9 \\
\mathrm{C}: 34-66,48.4\end{array}$ & $\begin{array}{l}\text { T: } 26 / 19 \\
\text { C: } 23 / 22\end{array}$ & $\begin{array}{l}\text { T: } 1-6,3.4 \\
\text { C: } 1-7,3.1\end{array}$ \\
\hline Xu et al. [17] & 2011 & $30 / 30$ & $\begin{array}{l}\mathrm{T}: 18-53,42.2 \\
\mathrm{C}: 16-58,45.3\end{array}$ & $\begin{array}{l}\text { T: } 18 / 12 \\
\text { C: } 20 / 10\end{array}$ & $\begin{array}{c}\mathrm{T}: 0.1-3 \\
\mathrm{C}: 0.1-3.2\end{array}$ \\
\hline Zhu [18] & 2010 & $45 / 45$ & $\begin{array}{l}\text { T: } 22-75,44.6 \\
\text { C: } 34-68,46.1\end{array}$ & $\begin{array}{l}\mathrm{T}: 20 / 25 \\
\mathrm{C}: 22 / 23\end{array}$ & $\begin{array}{l}\mathrm{T}: 0.1-0.4 \\
\mathrm{C}: 0.1-0.3\end{array}$ \\
\hline He et al. [19] & 2008 & $32 / 28$ & $\begin{array}{l}\text { T: } 16-61,32 \\
\text { C: } 17-59,31\end{array}$ & $\begin{array}{l}\text { T: } 19 / 13 \\
\text { C: } 16 / 12\end{array}$ & NR \\
\hline Xie et al. [20] & 2008 & $30 / 30$ & $\begin{array}{l}\text { T: } 22-61,38.5 \\
\text { C: } 19-65,42.1\end{array}$ & $\begin{array}{c}\text { T: } 22 / 8 \\
\text { C: } 20 / 10\end{array}$ & $\begin{array}{l}\mathrm{T}: 0.1-6,4.5 \\
\mathrm{C}: 0-10,4.7\end{array}$ \\
\hline Shu [21] & 2007 & $24 / 24$ & $\begin{array}{ll} & \text { T: } 36.2 \\
19-53 & \text { C: } 37.8\end{array}$ & $\begin{array}{l}\mathrm{T}: 20 / 4 \\
\mathrm{C}: 18 / 6\end{array}$ & $\begin{array}{l}\text { T: } 0.2 \\
\text { C: } 0.2\end{array}$ \\
\hline $\operatorname{Li}[22]$ & 2006 & $32 / 31$ & $\begin{array}{l}\mathrm{T}: 37.1 \\
\mathrm{C}: 37.3\end{array}$ & $\begin{array}{l}\mathrm{T}: 22 / 10 \\
\mathrm{C}: 17 / 14\end{array}$ & $\begin{array}{l}\text { T: } 0.1 \\
\text { C: } 0.1\end{array}$ \\
\hline He et al. [23] & 2003 & $121 / 117$ & NR & NR & NR \\
\hline $\mathrm{He}^{*}$ et al. [24] & 2003 & $312 / 104$ & $\begin{array}{l}\text { T: } 39.8 \\
\text { C: } 41.3\end{array}$ & $\begin{array}{l}\text { T: } 233 / 79 \\
\text { C: } 80 / 24\end{array}$ & $\begin{array}{l}\text { T: } 0.1^{\mathrm{a}}, 3.1^{\mathrm{ch}} \\
\mathrm{C}: 0.1^{\mathrm{a}}, 3.3^{\mathrm{ch}}\end{array}$ \\
\hline
\end{tabular}

T: trials group, C: control group. NR: no report. ${ }^{a}$ acute cholestatic hepatitis, ${ }^{\text {ch }}$ chronic cholestatic hepatitis.

“*” in Table 1 and Table 2 refers to another study different from the study of the same name and year' 


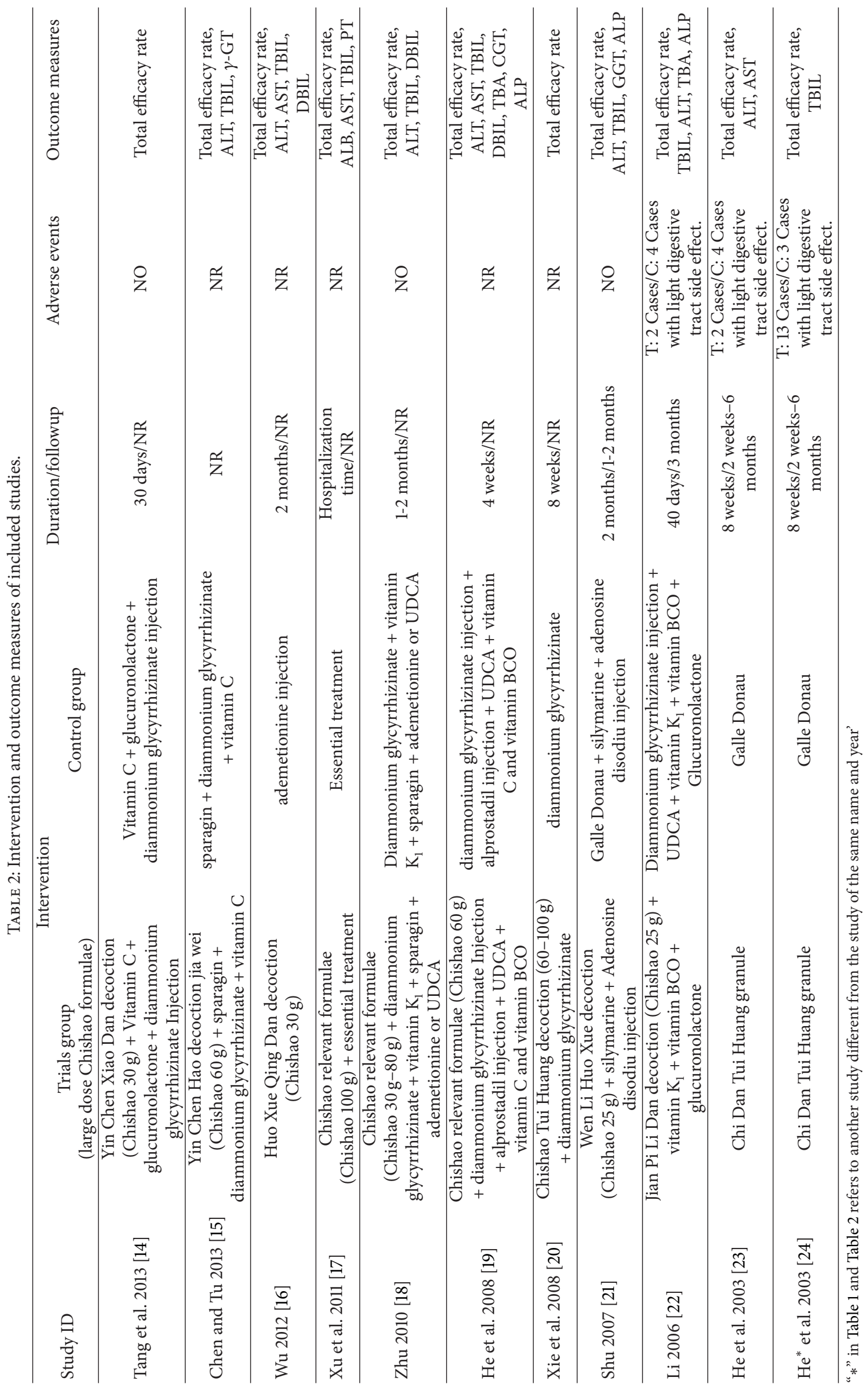




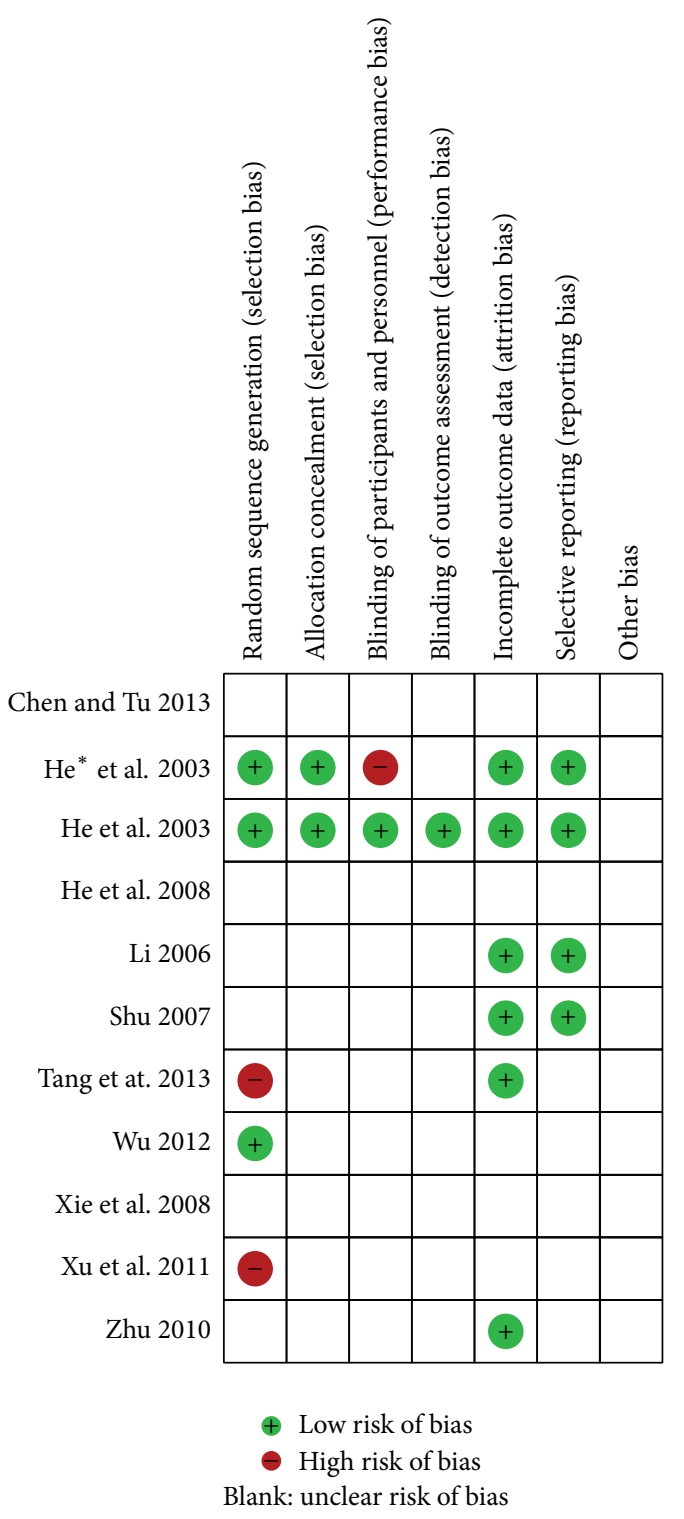

FIGURE 2: Methodological quality assessment for risk of bias for each included study.

3.2. Methodological Quality of Included Trials. According to Cochrane risk of bias estimation, randomized allocation of participants was mentioned in all trials; however, 3 trials reported randomized methods $[16,23,24]$ through number table and 2 through clinical sequence [14, 17]. Allocation concealment was performed in 2 of 11 articles $[23,24]$ and 1 mentioned blinding of participants and personnel and blinding of outcome assessment [23]. There were no incomplete outcome data or essential data missing in 6 reports $[14,18,21-$ $24]$, whereas others were unclear. Four articles mentioned systematic project in research [21-24] and others remained unclear (Figure 2).

\subsection{Outcome Measures}

3.3.1. Formulae with or without Essential Treatment versus Essential Treatment. Three of 11 articles described trials group with only Chishao formulae treatment, whereas the rest 8 RCTs reported combination treatment of Chishao formulae and essential drugs in trials group. No heterogeneity was displayed in both analyses and fixed effects model was used $(P>0.10)$. The results displayed $\mathrm{RR}$ with $95 \% \mathrm{CI}$ in single application and combinations were $1.23(1.13,1.34)$ and $1.43(1.29,1.58)$. It revealed that formulae with large dosage of Chishao did demonstrate an exact efficacy in cholestatic hepatitis treatment whether combined application or not (Figure 3).

3.3.2. Serum Indices of Cholestatic Hepatitis Pathology. Serum ALT level was measured in 6 articles whereas 4 reported AST. TBIL level, the direct index of cholestatic hepatitis, was described in 9 trials and 4 displayed serum DBIL level. Heterogeneity occurred in indices of ALT, AST, TBIL, 


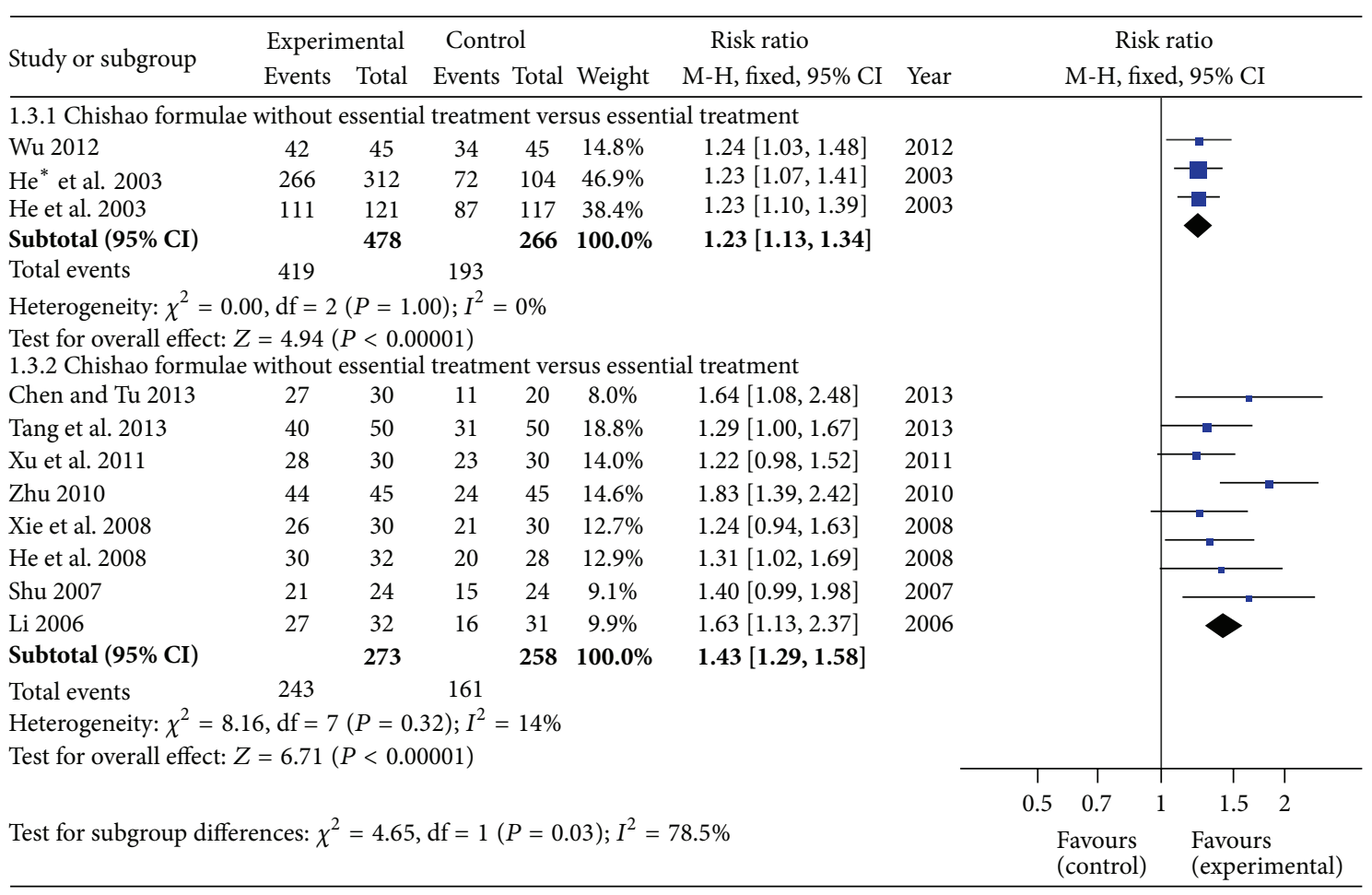

FIGURE 3: Forest plot of large dosage of Chishao relevant formulae with or without essential treatment versus essential treatment in patients with cholestatic hepatitis.

and DBIL $(P<0.10)$. Therefore, random effects model was adopted for analysis. The MDs with $95 \% \mathrm{CI}$ of serum ALT, AST, TBIL, and DBIL level were $-24.96(-34.02,-15$. $90),-22.84(-31.60,-14.08),-30.28(-42.55,-18.02)$, and $-15.13(-25.21,-5.04)$, respectively, indicating a significant pathologic indices decrease in trials group (Figure 4).

3.3.3. Efficacy Rate of Different Course of Disease. Cholestatic hepatitis was divided into two kinds of subtype according to the course of disease. In this study, the course of cholestatic hepatitis beyond 1 year was defined as long-term disease. Otherwise, cholestatic hepatitis within 1 year was classified to short term disease. There were 4 articles ascribed as shortterm subtype and 3 as long-term subtype. On the basis of fixed effects model $(P>0.10)$, both of the results showed a significant difference between trials and control. RR with $95 \%$ CI was $1.53(1.31,1.77)$ in shortterm and $1.23(1.08,1.40)$ in long term (Figure 5).

3.4. Adverse Events. Among 11 RCTs, 3 [14, 18, 21] reported no side effect occurring in clinical trials. Another 3 [2224] observed totally 28 cases of side effect presenting as light digestive tract symptom, but there was no causality assessment for this adverse event. In spite of no difference between trials and controls, systematic safety assessment of Chishao relevant formulae needed further investigation (Table 2).
3.5. Publication Bias. A funnel plot of large dosage of Chishao relevant formulae with essential treatment verses essential treatment in patients with cholestatic hepatitis was applied with RR as the $x$-axis and $\mathrm{SE}(\mathrm{RR})$ as the $y$-axis, respectively. The plot was symmetric, suggesting that the publication bias was little (Figure 6).

\section{Discussion}

Cholestasis is defined as an impairment of bile secretion and flow followed by a lack of bile in intestine and accumulation of potentially toxic BA in the liver and the systemic circulation. Cholestasis results in intrahepatic retention of cytotoxic BA which can thus lead to liver injury or liver fibrosis [27, 28]. In cholestatic hepatitis, serum TBIL and DBIL are regarded as the sensitive diagnosis indices of cholestatic hepatitis, meanwhile, ALT and AST represent the liver function along with disease. It is well known that a variety of definite factors including infection, drug abuse, autoimmunity, heredity, gestation, and operation cause intrahepatic cholestasis. As for the mechanism of cholestatic hepatitis, more focus is localized on oxidative stress [29], inflammatory response [30], and relevant hepatocyte transporters [31, 32]. However, a systematic explanation still remains vague. At present, UDCA is recognized as the specific and potent medicine for cholestasis [33] and often combined with glucocorticoid [34]. But for long duration therapy, glucocorticoid may lead to several side effects, meanwhile UDCA will not always 


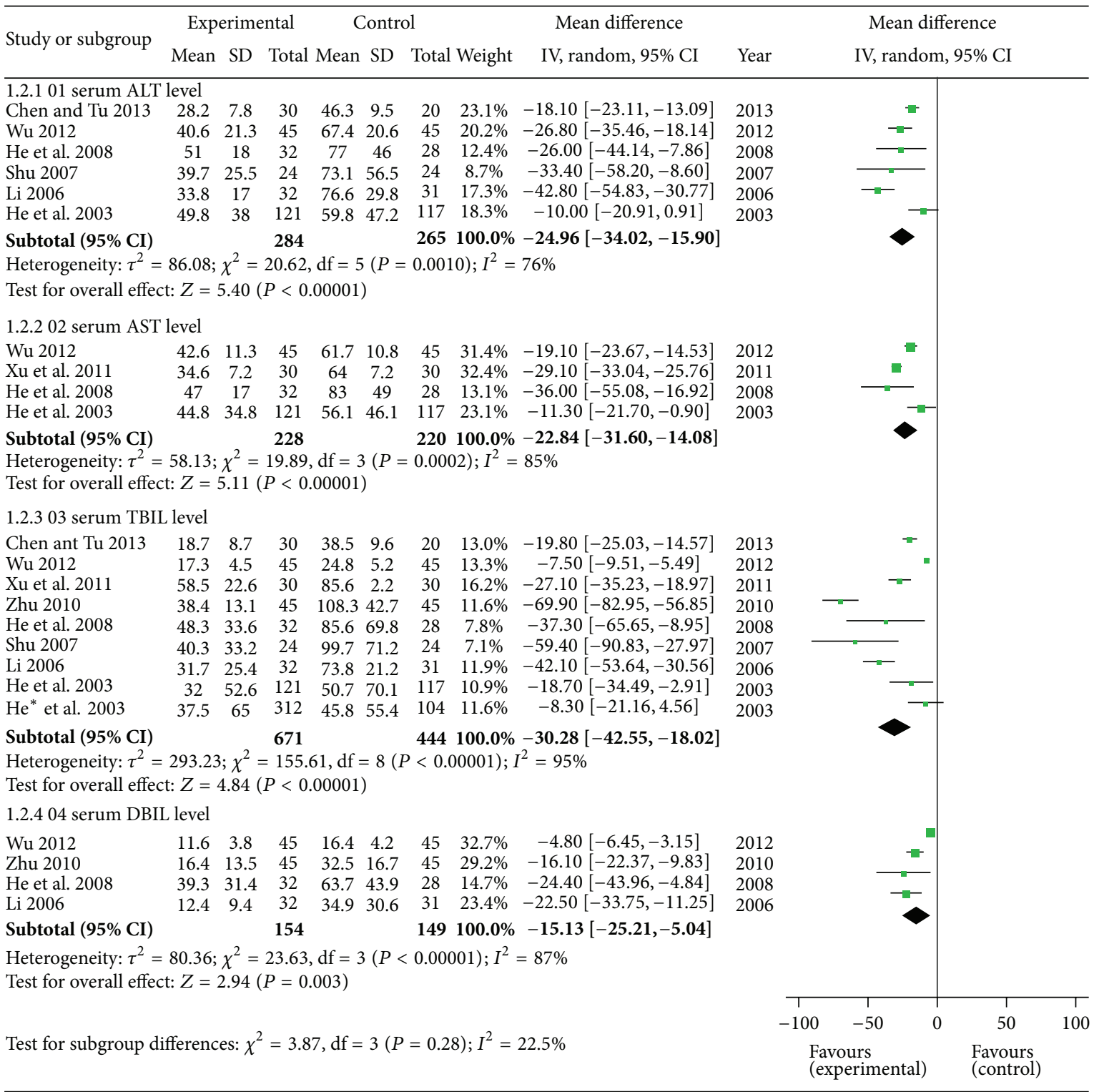

FIGURE 4: Forest plot of serum indices of cholestatic hepatitis pathology.

present stable efficacy. Transplantation, as the ultimate treatment, may result in severe immune rejection which is a devastating problem affecting quality of life. As one kind of alternative and complementary medicine, TCM shows the absolutely advantages in chronic diseases treatment. The integrated modulation to disease always involves in network style distributed to targets of molecules, tissues, and organs level $[35,36]$. There are a variety of herbs and formulae in TCM that represent obvious effect of removing jaundice. Although different herbs and formulae may display different mechanisms [37], the ultimate manifestation in macro view reaches the same.

Chishao serves as an important drug of TCM. The main components are monoterpene glycosides, flavonoids, tannins, and phenolic acids $[38,39]$. The herb and its active component consistently exhibit many pharmacological effects such as vasodilatation of thoracic aorta [40], antiallergic effect [41], liver protection [2], anti-inflammation, and immunoregulation [42]. However, application of large dosage of Chishao treating cholestatic hepatitis is a novel clinical practice in the recent 30 years [11]. Due to its unique efficacy, the therapeutic form is accepted by increasing practitioners. But from the safety and reasonable aspect, it brings about an essential question in TCM. Why can Chishao or even others as Rheum palmatum (Dahuang) and Coptis (Huanglian) be applied with such a large dosage? A recent study may reveal this answer related to the fact that different organic status represent different tolerance to drugs [43]. An experiment of Rheum palmatum extraction (RE) also indicates that RE, ranged certain dose, displays curative function in fibrosis animal but induces liver damage in normal [44]. Therefore, tolerance of pathologic status is relatively higher than 


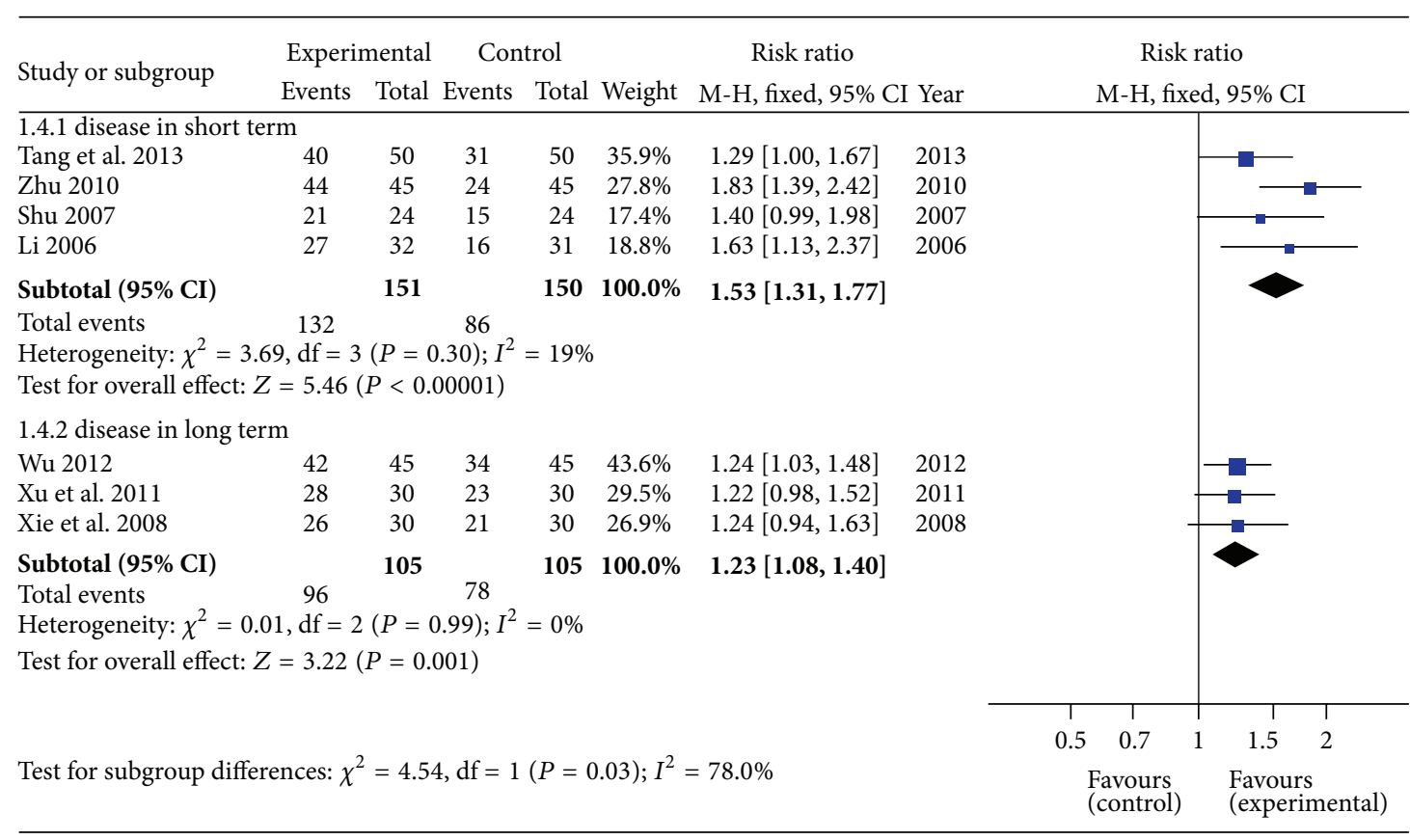

FIGURE 5: Forest plot of different course of cholestatic hepatitis.

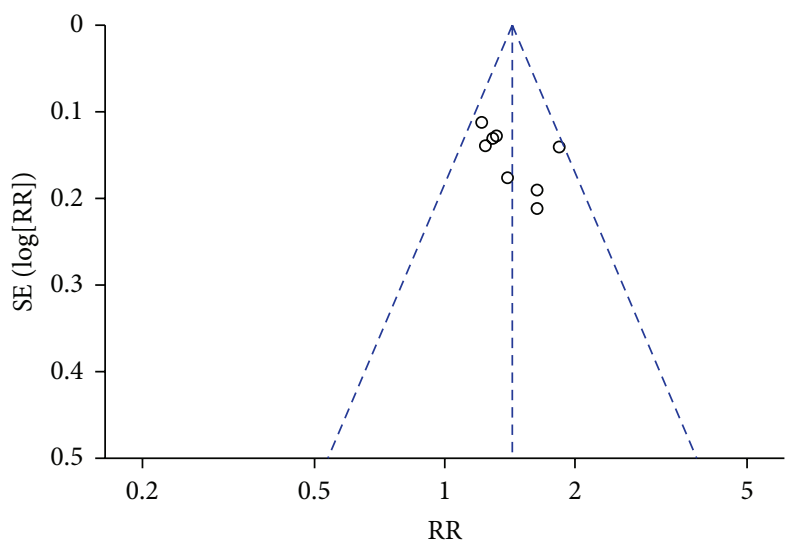

FIGURE 6: Funnel plot of large dosage of Chishao relevant formulae with essential treatment versus essential treatment in patients with cholestatic hepatitis.

normal one. Except that, the metabolism characteristic of Chishao is possibly another reason. The bioavailability of paeoniflorin and albiflorin in Chishao is also distinct, manifested as normal rats' bioavailability being lower compared with cholestasis hepatitis rats' [45]. Therefore, in specific pathologic status, large dosage of Chishao shall present the specific dose-effect relationship which will not be discovered in normal condition. According to both of the assumptions and examples, organic status may be the crucial and specific point to the therapy.

This systematic review included 11 randomized controlled trials and a total of 1275 participants. Large dosage of Chishao relevant formulae served as the key subject. It demonstrated a specific and potent effect for cholestasis hepatitis. As indices of hepatic pathology, the formulae led to a significant decrease in ALT, AST, TBIL, and DBIL compared with essential treatment. In addition, trials' serum level of TBIL presented the most dramatic decline, indicating a stronger removing jaundice effect than controls. Subgroup study of formulae without essential treatment manifested potent effect and formulae combined with essential treatment did also enhance the efficacy rate. The course of disease is another important factor which affects prognosis [46]. Different course of disease was investigated. The result illustrated that formulae were effective to the disease in short term or long term and acquired stronger effect than controls. Except Chishao, other herbs such as Dahuang, Yinchen, Danshen, and Gegen may also serve as important ingredients (see Supplementary Material available online at http://dx.doi.org/10.1155/2014/328152.) Effects of liver protection and jaundice removing existed in these herbs. Six articles also proved that there were no obvious severe advent 
events occurring in RCTs. Light digestive tract symptom was observed, but there was no causality assessment for this adverse event. From the systematic analysis, large dosage of Chishao relevant formulae exhibits a promising therapy for cholestasis hepatitis.

As the requirement for medical research in translational medicine, conventional drug discovery, as style of single target to animals, faces many challenges [47]. On the contrary, TCM presents abundant experiences in clinical treatment. Fundamental research can also be sustained or indicated through this treasure. Although clinical application is mature, the mechanism of Chishao relevant formulae's effect remains far from explicit. Therefore, further focus ought to be localized on internal relationship between the formulae and their effect through the model of "clinic-experiment-clinic."

\section{Conclusions}

Large dosage of Chishao relevant formulae may promote the curative efficacy of cholestasis hepatitis, which is a promising novel treatment approach. Considering being accepted by widespread practitioners, more rigorously designed multicenter, double-blind, randomized, and large-scale controlled trials are required.

\section{Conflict of Interests}

The authors declare that there is no conflict of interests regarding the publication of this paper.

\section{Authors' Contribution}

Xiao Ma and Ji wang contributed equally to this paper.

\section{Acknowledgments}

The current work was supported by the Major Projects of the National Science and Technology (no. 2012ZX10005010-002002) and the National Natural Science Foundation Project of China (nos. 81303120 and 81173571).

\section{References}

[1] F. Jin, D. Cheng, J. Y. Tao et al., "Anti-inflammatory and antioxidative effects of corilagin in a rat model of acute cholestasis," BMC Gastroenterology, vol. 13, no. 1, article 79, 2013.

[2] Y. Zhao, G. Zhou, J. Wang et al., "Paeoniflorin protects against ANIT-induced cholestasis by ameliorating oxidative stress in rats," Food and Chemical Toxicology, vol. 58, pp. 242-248, 2013.

[3] C. Zhu, H. Cao, X. Zhou et al., "Meta-analysis of the clinical value of danshen injection and huangqi injection in liver cirrhosis," Evidence-Based Complementary and Alternative Medicine, vol. 2013, Article ID 842824, 8 pages, 2013.

[4] D. Yang, G. J. Gao, K. Wei, X. J. Wang, and F. X. Sun, "Clinical analysis of cholestatic hepatitis characteristic," Chinese Journal of Clinicians, vol. 5, no. 24, pp. 7423-7424, 2011.

[5] D. Yang, F. X. Sun, G. J. Gao, K. Wei, and X. J. Wang, "Evaluation on herbal Yudan decoction on treatment of acute cholestatic hepatitis," Chinese Journal of Liver Diseases, vol. 5, no. 3, pp. 9-11, 2013.

[6] L. Zhang, G. Wang, W. Hou, P. Li, A. Dulin, and H. L. Bonkovsky, "Contemporary clinical research of traditional chinese medicines for chronic hepatitis B in china: an analytical review," Hepatology, vol. 51, no. 2, pp. 690-698, 2010.

[7] Y. Xia, H. Luo, J. P. Liu, and C. Gluud, "Phyllanthus species for chronic hepatitis B virus infection," Cochrane Database of Systematic Reviews, no. 4, Article ID CD008960, 2011.

[8] H. M. Liu, X. B. Wang, Y. J. Chang, and L. L. Gu, "Systematic review and meta-analysis of randomized controlled trials of integrative medicine therapy for treatment of chronic severe hepatitis," Journal of Chinese Integrative Medicine, vol. 10, no. 11, pp. 1211-1228, 2012.

[9] S. Li and B. Zhang, "Traditional Chinese medicine network pharmacology: theory, methodology and application," Chinese Journal of Natural Medicines, vol. 11, no. 2, pp. 110-120, 2013.

[10] S. Li, Z. Q. Zhang, L. J. Wu, X. G. Zhang, Y. D. Li, and Y. Y. Wang, "Understanding ZHENG in traditional Chinese medicine in the context of neuro-endocrine-immune network," IET Systems Biology, vol. 1, no. 1, pp. 51-60, 2007.

[11] C. B. Wang, J. P. He, Z. Y. Lei, and J. M. Chen, “Treating cholestatic hepatitis with large dosage of Chishao for cooling blood and promoting blood flow-13 cases report," Journal of Traditional Chinese Medicine, no. 6, pp. 30-32, 1983.

[12] J. P. He and C. B. Wang, "Treatment of severe cholestatic hepatitis by integrative medicines," Chinese Journal of Integrative Medicine, vol. 4, no. 1, pp. 18-21, 1998.

[13] S. S. Wei, Y. L. Zhao, F. J. Jiang et al., "Investigation of high dosage of Paeoniae Radix Rubra against ANIT-induced acute cholestasis hepatitis in rats," Chinese Journal of Experimental Traditional Medical Formulae, vol. 18, no. 12, pp. 151-155, 2012.

[14] X. Q. Tang, W. Q. Xu, and X. J. Cheng, "Yinchen Xiaodan method combined with western medicine in treatment of chronic hepatitis B with intrahepatic cholestasis randomized parallel controlled study," Journal of Practical Traditional Chinese Internal Medicine, vol. 27, no. 3, pp. 79-80, 2013.

[15] Z. Q. Chen and Y. Y. Tu, "Clinical observation of 30 cases of cholestatic hepatitis treating with ancient Jiawei prescription," Inner Mongol Journal of Traditional Chinese Medicine, vol. 12, pp. 18-19, 2013.

[16] Q. B. Wu, "control observation of Huoxue Qingdan decoction in treating chronic cholestatic hepatitis," Journal of Practical Traditional Chinese Internal Medicine, vol. 26, no. 8, pp. 49-51, 2012.

[17] C. J. Xu, W. S. Guo, and Y. F. Xie, "Clinical observation of 30 cases of severe cholestatic hepatitis treating with combined tratitional Chinsese medicine and western medicine," Modern Journal of Integrated Traditional Chinese and Western Medicine, vol. 20, no. 20, pp. 2531-2532, 2011.

[18] X. J. Zhu, "Clinical observation of cholestatic hepatitis treated with large dose Chishao," Clinical Journal of Traditional Chinese Medicine, vol. 22, no. 2, pp. 144-145, 2010.

[19] S. M. He, B. Huang, and X. Deng, "32 Cases of severe cholestatic hepatitis treated with Chi Yin Tui Huang decoction combined with western medicine," Shaanxi Journal of Traditional Chinese Medicine, vol. 29, no. 5, pp. 564-566, 2008.

[20] C. J. Xie, W. B. Ma, R. C. Li, and S. Y. Wang, "60 Cases of cholestatic hepatitis treated with Chishao Tuihuang decoction research," Capital Medicine, vol. 6, p. 49, 2008. 
[21] Z. E. Shu, Clinical Observation of Chronic Cholestatic Hepatitis Treated with Wen Li Huo Xue Decoction, Hubei University of Chinese Medicine, 2007.

[22] Y. F. Li, Clinical Observation of Chronic Viral Cholestatic Hepatitis After Treatment with Jian Pi Li Dan Decoction, Hubei University of Chinese Medicine, 2006.

[23] J. P. He, C. B. Wang, J. B. Ding et al., "Comparison of Chidan Tuihuang granule and Galle-Donau in the treatment of acute and chronic viral cholestatic hepatitis," Chinese Journal of New Drugs, vol. 12, no. 3, pp. 209-212, 2003.

[24] J. P. He, C. B. Wang, J. B. Ding et al., "Open comparative study of Chidan Tuihuang granule in treating acute and chronic viral cholestatie hepatitis," Chinese Journal of Integrated Traditional and Wetern Medicine, vol. 23, no. 7, pp. 498-500, 2003.

[25] Chinese Medical Association, "Viral hepatitis prevention and treatment programs," Infectious Disease Information, vol. 13, no. 4, pp. 141-150, 2000.

[26] X. Y. Zheng, Guidance for Clinical Research on New Drugs of TCM, China Medical Science Press, 2002.

[27] R. Poupon, O. Chazouilleres, and R. E. Poupon, "Chronic cholestatic diseases," Journal of Hepatology, vol. 32, no. 1, supplement, pp. 129-140, 2000.

[28] S. Kakizaki, D. Takizawa, H. Tojima, N. Horiguchi, Y. Yamazaki, and M. Mori, "Nuclear receptors CAR and PXR; therapeutic targets for cholestatic liver disease," Frontiers in Bioscience, vol. 16, no. 7, pp. 2988-3003, 2011.

[29] S. Anthérieu, P. B. Azzi, J. Dumont et al., "Oxidative stress plays a major role in chlorpromazine-induced cholestasis in human HepaRG cells," Hepatology, vol. 57, no. 4, pp. 1518-1529, 2013.

[30] K. Allen, H. Jaeschke, and B. L. Copple, "Bile acids induce inflammatory genes in hepatocytes: a novel mechanism of inflammation during obstructive cholestasis," The American Journal of Pathology, vol. 178, no. 1, pp. 175-186, 2011.

[31] A. Geier, M. Wagner, C. G. Dietrich, and M. Trauner, "Principles of hepatic organic anion transporter regulation during cholestasis, inflammation and liver regeneration," Biochimica et Biophysica Acta-Molecular Cell Research, vol. 1773, no. 3, pp. 283-308, 2007.

[32] D. Keppler, "Cholestasis and the role of basolateral efflux pumps," Zeitschrift für Gastroenterologie, vol. 49, no. 12, pp. 1553-1557, 2011.

[33] H. Buryova, K. Chalupsky, O. Zbodakova et al., "Liver protective effect of ursodeoxycholic acid includes regulation of ADAM17 activity," BMC Gastroenterology, vol. 13, no. 1, article 155, 2013.

[34] F. Arenas, I. Hervias, M. Úriz, R. Joplin, J. Prieto, and J. F. Medina, "Combination of ursodeoxycholic acid and glucocorticoids upregulates the AE2 alternate promoter in human liver cells," Journal of Clinical Investigation, vol. 118, no. 2, pp. 695-709, 2008.

[35] Z. Fang, B. Lu, M. Liu et al., "Evaluating the pharmacological mechanism of Chinese medicine Si-Wu-Tang through multilevel data integration," PLoS ONE, vol. 8, no. 11, Article ID e72334, 2013.

[36] J. Liu, M. Pei, C. Zheng et al., "A systems-pharmacology analysis of herbal medicines used in health improvement treatment: predicting potential new drugs and targets," Evidence-Based Complementary and Alternative Medicine, vol. 2013, Article ID 938764, 17 pages, 2013.

[37] A. Zhang, H. Sun, S. Qiu, and X. Wang, "Advancing drug discovery and development from active constituents of Yinchenhao Tang, a famous traditional Chinese medicine formula,"
Evidence-Based Complementary and Alternative Medicine, vol. 2013, Article ID 257909, 6 pages, 2013.

[38] Y. Z. Wang, W. S. Feng, R. B. Shi, B. Liu, and Y. Y. Jiang, "Monoterpenes of Paeonia lactiflora Pall," Chinese Pharmaceutical Journal, vol. 43, no. 9, pp. 669-671, 2008.

[39] Y. Y. Yang, G. Zhou, X. K. Ma et al., "Research progress of Paeonia lactiflora Pall," Herald of Medicine, vol. 27, no. 1, pp. 6769, 2008.

[40] S. N. Jin, J. F. Wen, T. T. Wang, D. G. Kang, H. S. Lee, and K. W. Cho, "Vasodilatory effects of ethanol extract of Radix Paeoniae Rubra and its mechanism of action in the rat aorta," Journal of Ethnopharmacology, vol. 142, no. 1, pp. 188-193, 2012.

[41] B. Lee, Y. W. Shin, E. A. Bae et al., "Antiallergic effect of the root of Paeonia lactiflora and its constituents paeoniflorin and paeonol," Archives of Pharmacal Research, vol. 31, no. 4, pp. 445450, 2008.

[42] D. Y. He and S. M. Dai, "Anti-inflammatory and immunomodulatory effects of Paeonia lactiflora Pall., a traditional Chinese herbal medicine," Frontiers in Pharmacology, vol. 2, article 10, 2011.

[43] É. Bourbonnais, V. Raymond, C. Éthier et al., "Liver fibrosis protects mice from acute hepatocellular injury," Gastroenterology, vol. 142, no. 1, pp. 130.e4-139.e4, 2012.

[44] J. B. Wang, H. P. Zhao, Y. L. Zhao et al., "Hepatotoxicity or hepatoprotection? pattern recognition for the paradoxical effect of the chinese herb Rheum palmatum L. in treating rat liver injury," PLoS ONE, vol. 6, no. 9, Article ID e24498, 2011.

[45] F. Jiang, Y. Zhao, J. Wang et al., "Comparative pharmacokinetic study of paeoniflorin and albiflorin after oral administration of Radix Paeoniae Rubra in normal rats and the acute cholestasis hepatitis rats," Fitoterapia, vol. 83, no. 2, pp. 415-421, 2012.

[46] Y. F. Liaw, "Impact of therapy on the outcome of chronic hepatitis B," Liver International, vol. 33, supplement 1, pp. 111115, 2013.

[47] F. M. Marincola, "Translational medicine: a two-way road," Journal of Translational Medicine, vol. 1, no. 1, article 1, 2003. 


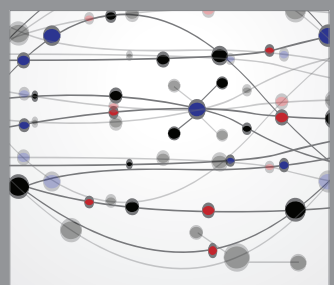

The Scientific World Journal
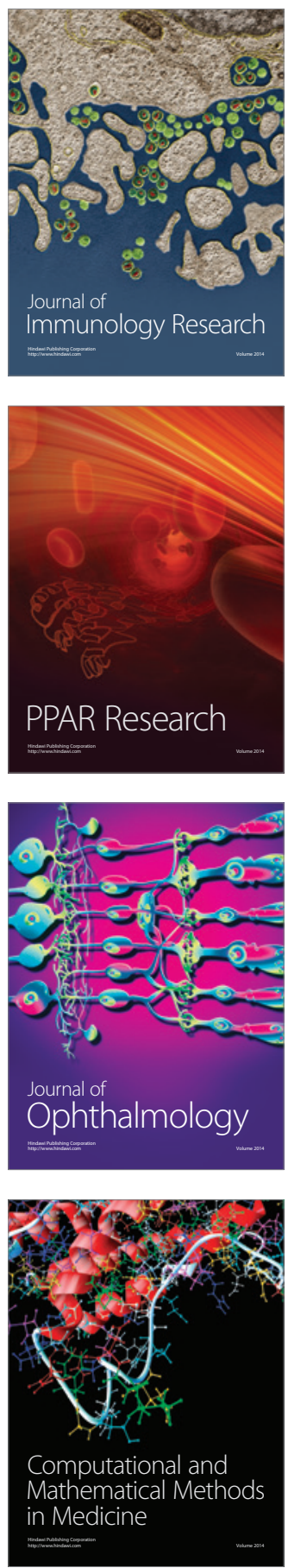

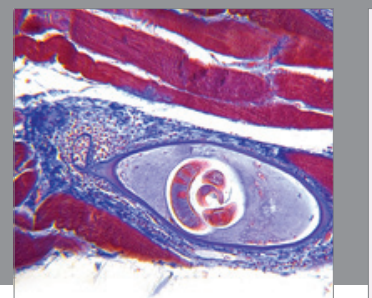

Gastroenterology

Research and Practice
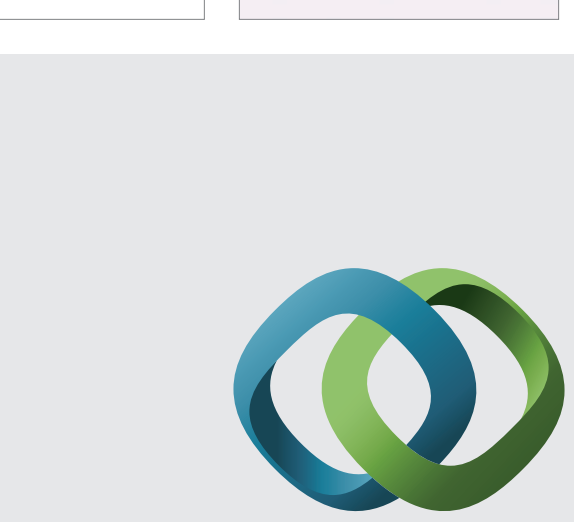

\section{Hindawi}

Submit your manuscripts at

http://www.hindawi.com
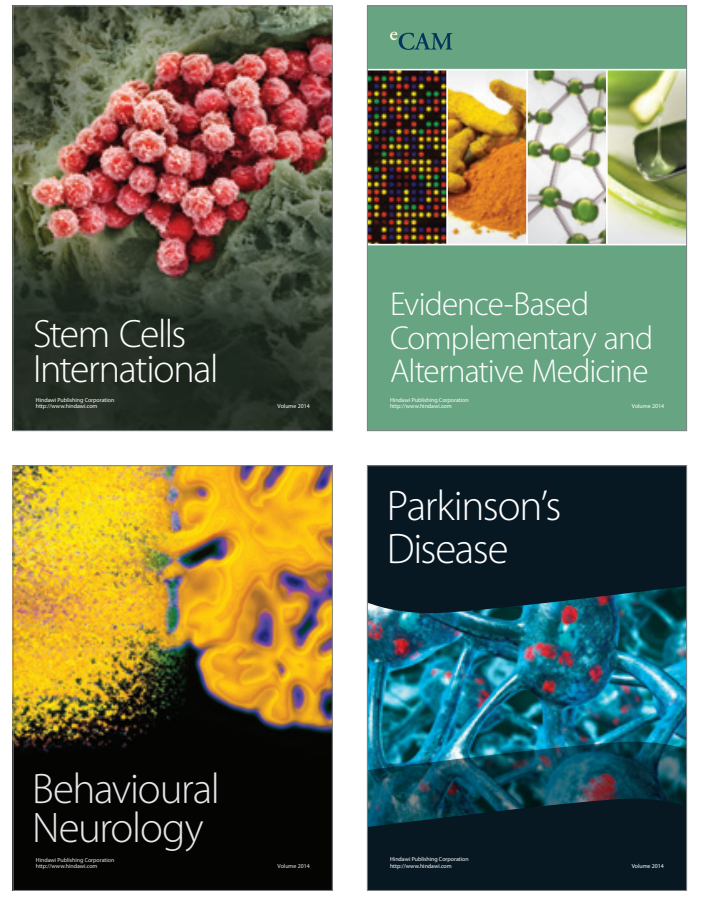
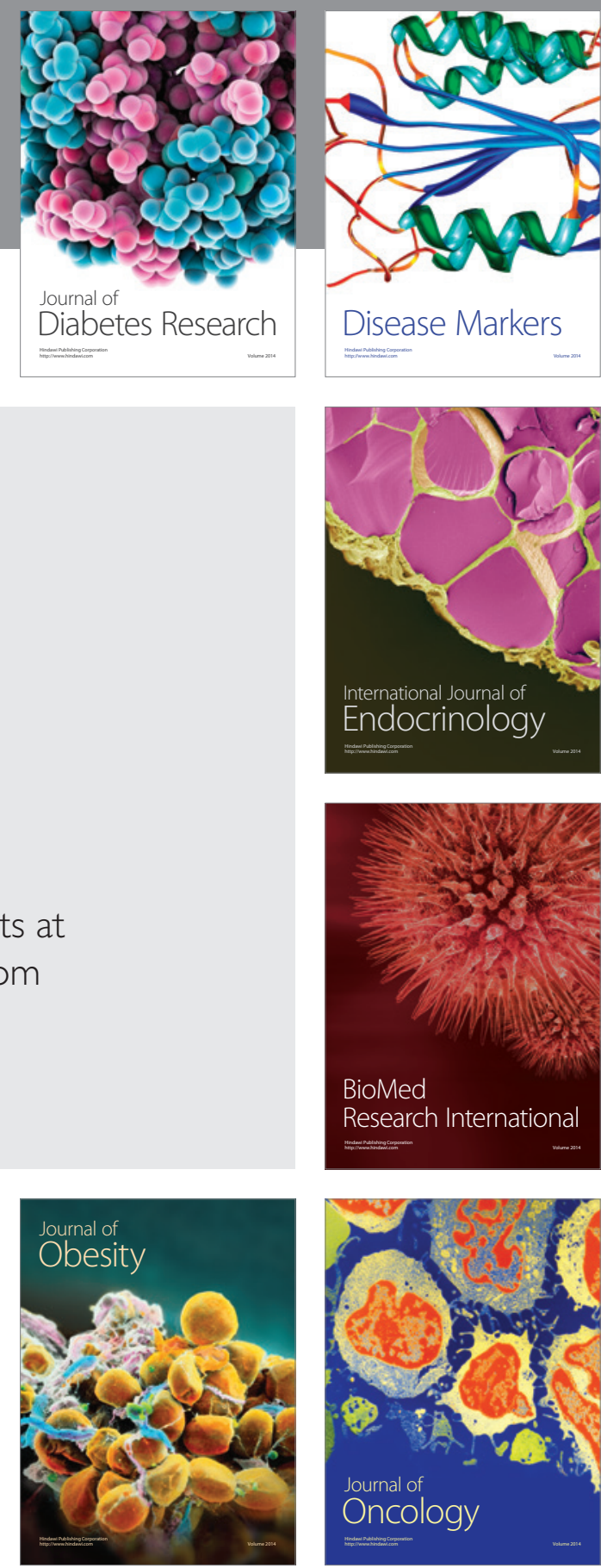

Disease Markers
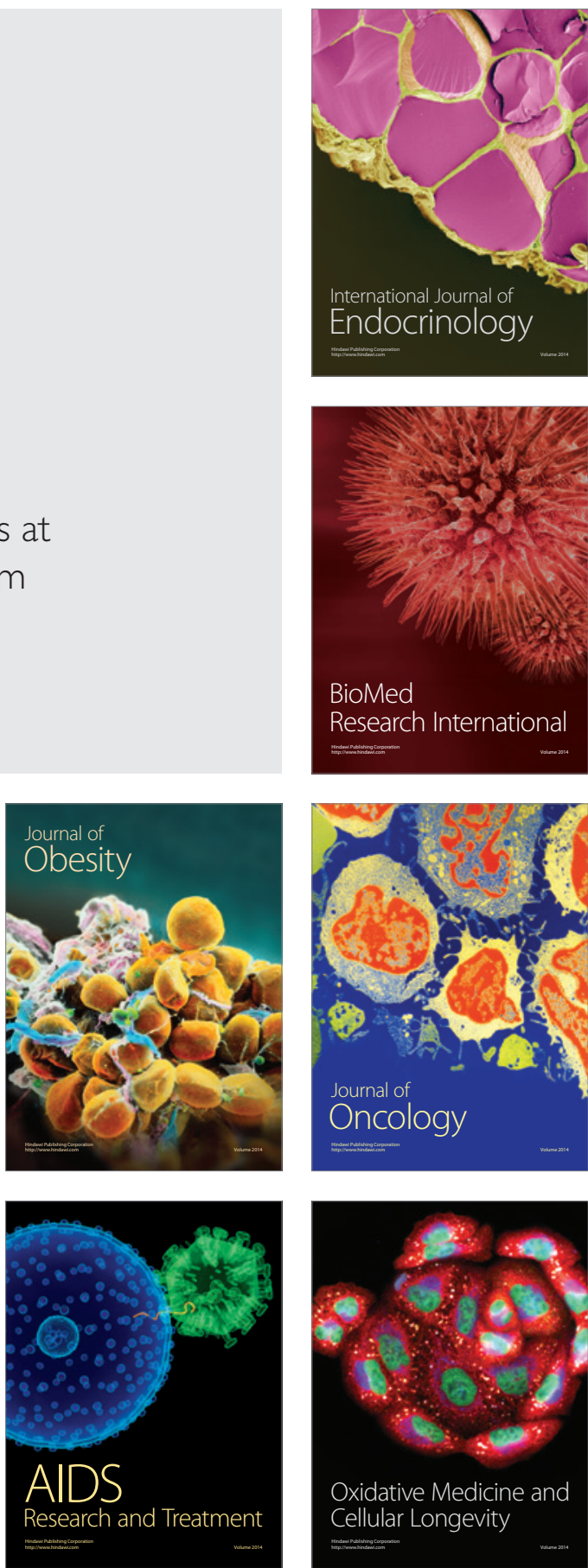